\title{
NUMERICAL STUDY OF RESERVOIR COOLING BY MEANS OF PELTIER EFFECT
}

\author{
Farias, R. M. ${ }^{1}$, Dos Santos, E.D. ${ }^{2}$, Isoldi, L. A. ${ }^{3}$, Rocha, L.A.O., \\ ${ }^{1}$ School of Engineering, Universidade Federal do Rio Grande, Italia Avenue, km 8, \\ Cx.P. 474, Rio Grande, RS, Brasil, 96201-900. \\ ${ }^{2}$ Department of Mechanical Engineering, Universidade Federal do Rio Grande do \\ Sul, Sarmento Leite Street, 425, Porto Alegre, RS, Brasil, 90050-170. \\ ${ }^{3}$ Program of Post-graduation in Computational Modeling, Universidade Federal do \\ Rio Grande, Italia Avenue, km 8, Cx.P. 474, Rio Grande, RS, Brasil, 96201-900.
}

\begin{abstract}
The present work studies numerically and experimentally the water cooling process by means of natural convection inside a closed reservoir. The cooling process is performed by Peltier or Thermoelectric effect. The purpose here is to obtain the thermal gradient inside the reservoir and to search for the best point where the coldest water can be extracted from the reservoir, which can be considered a geometric optimization of the device thermal design. The analyzed flow is incompressible, laminar, transient and three-dimensional. The Boussinesq approximation is employed for the treatment of buoyancy forces. For the numerical approach of the flow, the mass, momentum and energy conservation equations are solved by a commercial package based on the finite volume method (FLUENT ${ }^{\circledR}$ ). The temperature field as function of time obtained by numerical simulations is confronted with the experimental data. The numerical results estimated satisfactorily the transient thermal behavior predicted by laboratory experiments.
\end{abstract}

Keywords: Peltier Effect; Natural Convection; Transient Flow; Numerical Analysis; Experimental Analysis.

* Corresponding author: laorocha@gmail.com Phone: +5553 32336883 


\section{INTRODUÇÃO}

Atualmente, o homem procura desenvolver novas tecnologias para aumentar a eficiência e diminuir custos em equipamentos da área térmica, sejam eles para fins de refrigeração ou aquecimento, além de garantir uma melhor qualidade de vida para quem os utiliza.

A tecnologia mais amplamente utilizada nesta área é a do ciclo de refrigeração por compressão padrão. Mas atualmente ela vem perdendo espaço para a refrigeração eletrônica, quando se trata de aplicações de pequeno porte, nas áreas industrial, automobilística, militar, bens de consumo, dentre outras.

Alguns exemplos destas aplicações são: bebedouros, aquecedores/resfriadores de bancos, minigeladeiras, resfriamento de microprocessadores, e até em trocadores de calor dentro de submarinos (Riberi, 2004).

A refrigeração eletrônica baseia-se no efeito Peltier, no qual ao se forçar uma corrente elétrica por uma junção de dois materiais diferentes, ocorre um efeito térmico: um lado da junção esfria e o outro esquenta. Portanto, há transferência de calor de um lado da junção para o outro. Utilizam este efeito os assim chamados módulos Peltier, também conhecidos como pastilhas termoelétricas.

Neste trabalho será feito um estudo dos módulos de refrigeração dos bebedouros. Mais precisamente, este estudo será feito sobre o fenômeno de convecção natural que acontece dentro do reservatório, enquanto a água é resfriada da temperatura ambiente até uma determinada temperatura mais baixa.

Este estudo será feito através de simulações numéricas em uma geometria tridimensional do reservatório, que serão feitas no software FLUENT ${ }^{\circledR}$ (FLUENT, 2007), o qual discretiza as equações a partir do método de volumes finitos. O objetivo deste trabalho é validar uma modelagem numérica de um sistema físico de convecção natural interna com dados experimentais e, por conseqüência, determinar o gradiente de temperaturas dentro do reservatório analisado após um determinado tempo de funcionamento do equipamento.

Utilizando as equações de conservação da massa, da energia e da quantidade de movimento, e tendo todos os parâmetros devidamente ajustados, este software é capaz de calcular a distribuição de temperatura e de outras propriedades do fluido e, através de uma comparação com os resultados experimentais, é possível determinar se o modelo é válido ou não. 
O processo de resfriamento da água dentro do reservatório é considerado tridimensional, laminar, incompressível, em regime transiente e com todas propriedades constantes, exceto a massa específica, que varia segundo a aproximação de Boussinesq (Bejan, 2004).

Conforme o equipamento começa a funcionar, o módulo Peltier começa a trabalhar como a bomba de calor do sistema, proporcionando uma troca térmica entre a água que se encontra no reservatório e o ambiente externo, por meio dos dissipadores de calor interno e externo (Masterfrio, 2008). Com a variação da temperatura, também ocorre à variação da densidade da água, dando início ao fenômeno de convecção natural, com a água sendo resfriada da temperatura ambiente até uma determinada temperatura mais baixa, geralmente em torno de $6^{\circ} \mathrm{C}$. Uma ilustração do equipamento é apresentada na FIGURA 1.

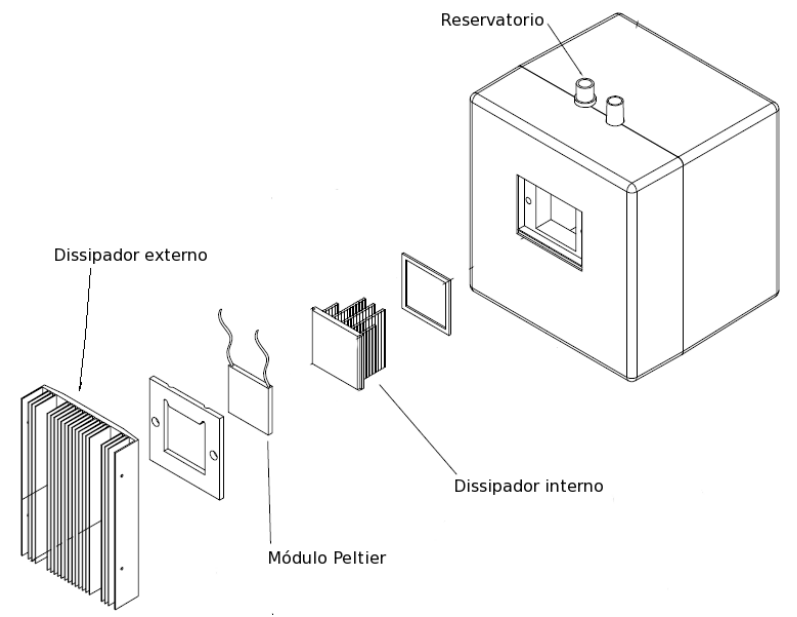

Figura 1 - Vista explodida da unidade de resfriamento, composta pelos dissipadores interno e externo, o módulo Peltier e o reservatório

\section{EFEITO TERMOELÉTRICO}

O efeito Termoelétrico consiste na conversão direta de diferenças de temperatura em tensão elétrica e vice-versa. Um dispositivo termoelétrico gera uma tensão quando há uma temperatura diferente em cada um de seus lados. Inversamente, quando uma tensão é aplicada a ele, cria-se uma diferença de temperatura, conhecida como efeito Peltier.

Este efeito pode ser usado para gerar eletricidade, para medir temperatura, para esfriar os objetos, ou para aquecê-los. Como a direção de aquecimento e 
arrefecimento é determinada pelo sinal da tensão aplicada, dispositivos termoelétricos são considerados controladores de temperatura muito convenientes.

Tradicionalmente, o termo efeito Termoelétrico ou Termoeletricidade engloba três efeitos identificados separadamente: o efeito Seebeck, o efeito Peltier e o efeito Thomson. Em muitos livros didáticos, o efeito termoelétrico também pode ser chamado de efeito Peltier-Seebeck. Esta separação decorre da descoberta independente do físico francês Jean Charles Athanase Peltier e do físico estonianogermânico Thomas Johann Seebeck.

O efeito Joule, no qual calor é gerado quando uma tensão elétrica é aplicada através de um material resistente, tem alguma relação, mas não é geralmente chamado de efeito termoelétrico. Os efeitos Peltier-Seebeck e Thomson podem, em princípio, ser termodinamicamente reversíveis (DiSalvo, 1999), ao contrário do que ocorre no efeito Joule.

$\mathrm{Na}$ convecção natural, o movimento do fluido se deve ao empuxo no interior do mesmo. De fato, o empuxo se deve à presença de um gradiente da massa específica do fluido que induz uma força de campo que atua como o principal mecanismo motriz do escoamento.

$\mathrm{Na}$ prática, a força de volume é usualmente a força gravitacional. Existem também muitas formas nas quais o gradiente de massa específica pode aparecer em um fluido, mas nas condições mais comuns, se deve à presença de um gradiente de temperatura.

Assim como na convecção forçada, as equações que descrevem a transferência de quantidade de movimento e de energia, na convecção natural, originam-se dos princípios de conservação pertinentes (massa, quantidade de movimento e energia). Além disso, os processos específicos relacionados à convecção natural são muito semelhantes aos que dominam a convecção forçada. As forças de inércia e as forças viscosas permanecem importantes, e também a transferência de energia por advecção e difusão. A diferença principal entre os dois tipos de escoamentos está no papel principal do empuxo. É esta força que, de fato, sustenta o escoamento (Incropera et al., 2008).

Neste trabalho é admitido que o escoamento é incompressível e o efeito da massa específica variável sobre o empuxo é abordada pelo produto de um coeficiente de expansão térmica e a diferença de temperaturas local e em um estado de referência. No que diz respeito ao acoplamento entre o campo de velocidades e o 
de temperaturas, é inserido um termo de forças de campo na equação de conservação da quantidade de movimento que depende do campo de temperaturas, e a aproximação de Boussinesq é empregada para expressar a variação de massa específica em função do campo de temperaturas (Bejan, 2004).

\section{MODELO MATEMÁTICA}

Nesta secção são descritas de forma resumida as equações gerais que expressam os fenômenos físicos envolvidos, e o método a ser utilizado para a resolução do problema. Na modelagem a ser realizada com o software FLUENT ${ }^{\circledR}$, são necessários dados referentes à geometria, as condições de contorno do escoamento, além das propriedades termofísicas do fluido de trabalho.

No domínio em questão, serão resolvidas as equações de conservação da massa (equação da continuidade); da quantidade de movimento; e a equação da energia são apresentadas, respectivamente, em Bejan (2004):

$$
\begin{aligned}
& \frac{\partial \rho}{\partial t}+\nabla \cdot(\rho \vec{v})=0 \\
& \frac{\partial}{\partial t}(\rho \vec{v})+\nabla \cdot(\rho \vec{v} \vec{v})=-\nabla P+\nabla \bar{\tau}+\rho \vec{g} \\
& \frac{\partial}{\partial t}(\rho E)+\nabla \cdot(\vec{v}(\rho E+p))=\nabla \cdot\left(k \nabla T-\sum_{j} h_{j} \vec{J}_{j}+\left(\bar{\tau}_{\text {eff }} \cdot \vec{v}\right)\right)+S_{h}
\end{aligned}
$$

onde $\rho$ é a massa específica do fluido $\left(\mathrm{kg} / \mathrm{m}^{3}\right)$, $t$ é o tempo (s), $v$ é o vetor velocidade $(\mathrm{m} / \mathrm{s}), P$ é a pressão $\left(\mathrm{N} / \mathrm{m}^{2}\right), \tau$ é a tensão de cisalhamento $\left(\mathrm{N} / \mathrm{m}^{2}\right), g$ é a aceleração do campo gravitacional $\left(\mathrm{m} / \mathrm{s}^{2}\right)$, E é a energia interna específica $(\mathrm{kJ} / \mathrm{kg}), S_{\mathrm{h}}$ é um termo fonte de energia. Além destes termos, o segundo termo do lado direito da equação da energia, Eq. (3), refere-se à transferência de energia por difusão das espécies e o terceiro termo está relacionado com a dissipação viscosa. Ambos os termos são desprezados no presente trabalho.

Além disso, para efetuar os cálculos em relação à variação de massa específica, será utilizada a aproximação de Boussinesq. Essa aproximação considera a densidade do fluido como função apenas da temperatura (FLUENT, 2007). Esta hipótese trata a densidade como uma constante em todas as equações, exceto no termo de empuxo na equação do momento.

$$
\left(\rho-\rho_{0}\right) g \approx-\rho_{0} \beta\left(T-T_{0}\right) g
$$


onde $\rho_{0}$ é a massa específica na temperatura de referência do fluido $\left(\mathrm{kg} / \mathrm{m}^{3}\right), T_{0}$ é a temperatura de referência $\left({ }^{\circ} \mathrm{C}\right.$ ou $\left.\mathrm{K}\right)$ e $\beta$ é o coeficiente de expansão térmica $\left(\mathrm{K}^{-1}\right)$. A Eq. (4) é obtida usando a aproximação de Boussinesq $\rho=\rho_{0}(1-\beta) \Delta T$ para eliminar $\rho$ no termo de empuxo. Essa aproximação é bem precisa desde que as mudanças na densidade sejam pequenas. Mais especificamente, a aproximação de Boussinesq é válida quando $\beta\left(T-T_{0}\right)$ é muito menor que 1 (Bejan, 2004).

O fluido a ser resfriado dentro do reservatório é a água. O dissipador interno, que é responsável pela troca de calor entre a água e o ambiente externo do reservatório, é feito de alumínio e todas suas propriedades são constantes. As paredes do reservatório não trocam calor com o ambiente (perfeitamente isoladas) e todo o calor retirado da água é extraído pela base do dissipador interno.

\section{MODELO NUMÉRICO}

O software comercial empregado para a solução das equações de conservação que governam o problema baseia-se no método de volumes finitos. Este método baseia-se no balanço de massa, quantidade de movimento e energia em um volume discretizado. Além disso, o método é oriundo do método de diferenças finitas e tem sido extensivamente utilizado na solução de problemas de mecânica dos fluidos e transferência de calor.

Neste método, as variáveis primárias em um domínio contínuo passam a ser descritas de forma discreta de forma que o conjunto de equações diferenciais que governa o problema seja substituído por um sistema de equações algébricas que são resolvidas numericamente.

Para a discretização do domínio e para a resolução do problema foram utilizados, respectivamente, os softwares GAMBIT ${ }^{\circledR}$ e FLUENT $^{\circledR}$ (FLUENT, 2007). Para estas simulações, foi utilizada uma geometria com 82.387 células, valor que foi definido a partir do teste de independência de malha. Nas FIGURAS 2 e 3 podem ser vistos o domínio computacional gerado e a discretização deste, respectivamente, que são gerados no sotware GAMBIT ${ }^{\circledR}$. 


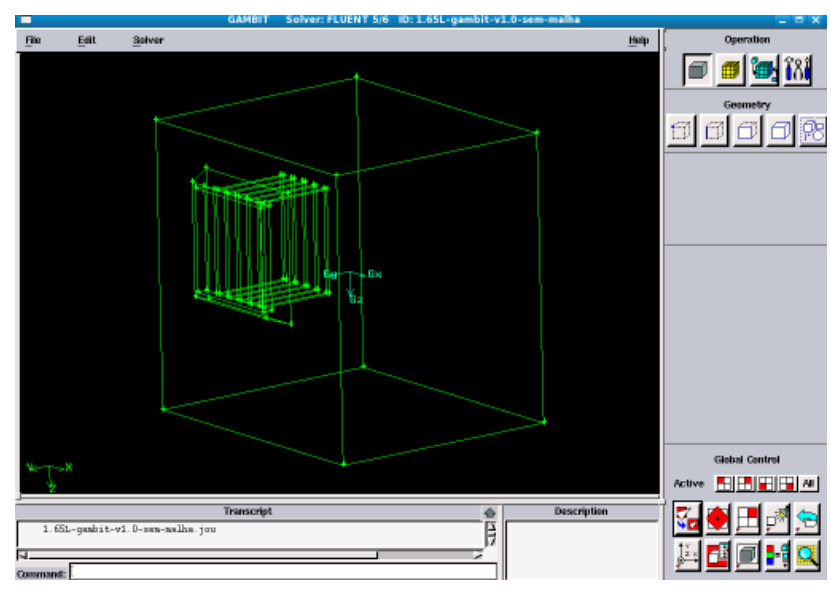

Figura 2 - Visualização da geometria do reservatório e do dissipador interno, no software GAMBIT ${ }^{\circledR}$.

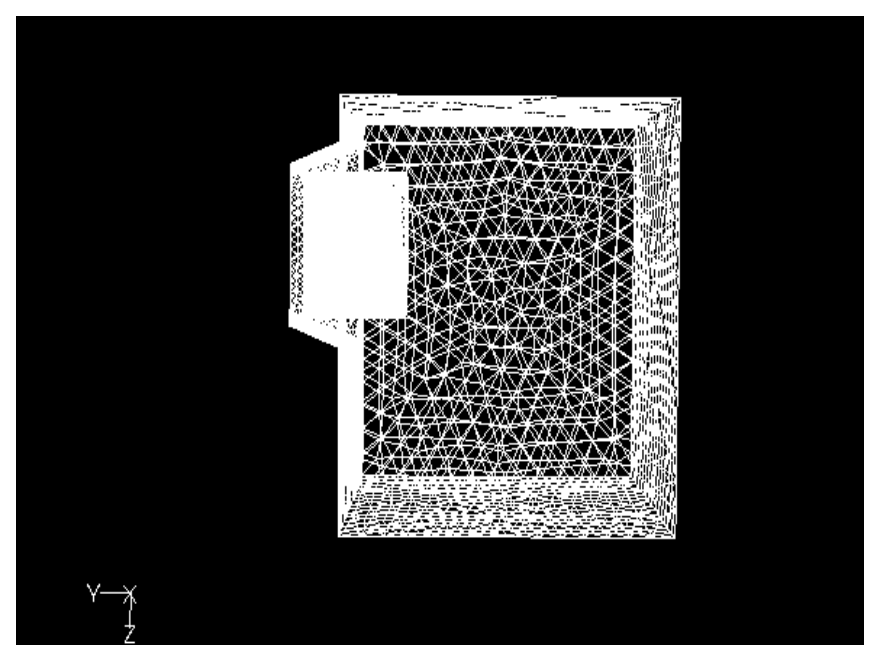

Figura 3 - Vista lateral da malha gerada no reservatório, com o dissipador localizado na região superior-esquerda.

Foram feitas duas simulações: uma com um fluxo de $12.000 \mathrm{~W} / \mathrm{m}^{2}$; e outra com $10.000 \mathrm{~W} / \mathrm{m}^{2}$, pois foi necessário corrigir este valor devido à eficiência da pastilha termoelétrica.

Na Tabela 1 constam alguns dados importantes utilizados para as simulações realizadas.

Tabela 1 - Propriedades e outras variáveis necessárias para as simulações.

\begin{tabular}{cc}
\hline Variáveis & Valores \\
\hline Fluxo de calor & $q_{1}=-12000 \mathrm{~W} / \mathrm{m}^{2} \quad q_{2}=-10000 \mathrm{~W} / \mathrm{m}^{2}$ \\
\hline Massa específica da água $(\rho)$ & $998,2 \mathrm{~kg} / \mathrm{m}^{3}$ \\
\hline Coeficiente de expansão térmica da água $(\beta)$ & $0,000276 \mathrm{~K} \mathrm{~K}^{-1}$ \\
\hline Temperatura inicial & $27^{\circ} \mathrm{C}$ \\
\hline
\end{tabular}




\section{RESULTS}

Foi requisitado a um laboratório de controle de qualidade um teste que simulasse 0 uso do equipamento. $O$ teste prossegue da seguinte maneira: 0 reservatório do equipamento é preenchido por completo com água, a uma temperatura de aproximadamente $27^{\circ} \mathrm{C}$. Então, o equipamento é ligado e começa a contagem de tempo. Devido ao efeito Peltier na pastilha termoelétrica, o calor começa a ser retirado do volume de água através do dissipador interno e é transferido para o dissipador externo que então libera este calor no ambiente. Quando a temperatura da água se aproxima de $5^{\circ} \mathrm{C}$, o que leva de duas a três horas, o teste é encerrado.

Esta temperatura de $5^{\circ} \mathrm{C}$ é usada como referência, uma vez que, devido à natureza da água, que atinge sua densidade máxima a aproximadamente $4^{\circ} \mathrm{C}$. Ao atingir este valor, a água no fundo do reservatório, onde se encontra o ponto de medição, continua a $4^{\circ} \mathrm{C}$ enquanto a água acima deste ponto continua baixando sua temperatura. Esse fenômeno é chamado de inversão térmica da água.

A FIGURA 4 ilustra o campo de temperaturas da água em função do tempo, observa-se que a temperatura da água chega a $6^{\circ} \mathrm{C}$ com aproximadamente 170 minutos de funcionamento, o que significa pouco mais do que duas horas e meia. Percebe-se que a temperatura da água no fundo do reservatório estabiliza neste valor. No entanto, o experimento indica o início do processo de congelamento da água na parte superior do reservatório, oriundo do processo de inversão térmica.

Utilizando os gráficos gerados pelo sistema de medição do laboratório, foi possível comparar os resultados experimentais com os resultados das simulações, resultados estes que são retirados aproximadamente do mesmo ponto da geometria onde é feita a medição.

Nas FIGURAS 5 e 6 é possível visualizar a evolução da temperatura ao longo do tempo no reservatório da simulação.

Na FIGURA 5 observa-se que para a predição a partir da simulação numérica o reservatório atingiu $6^{\circ} \mathrm{C}$ após 120 minutos. Apesar da diferença, o gráfico mostra a mesma tendência de curva ao efetuar o resfriamento.

Após verificar os dados do fabricante da pastilha termoelétrica, tomou-se a decisão de efetuar um ajuste no fluxo de calor, resultando no fluxo $q_{2}$ igual a 10.000 $\mathrm{W} / \mathrm{m} 2$. O resultado pode ser visto na FIGURA 6. 
Com este fluxo, a simulação do reservatório levou quase 140 minutos, ficando mais próximo do resultado experimental. É possível comparar todos os resultados em apenas um gráfico, para se obter um melhor entendimento da validação.

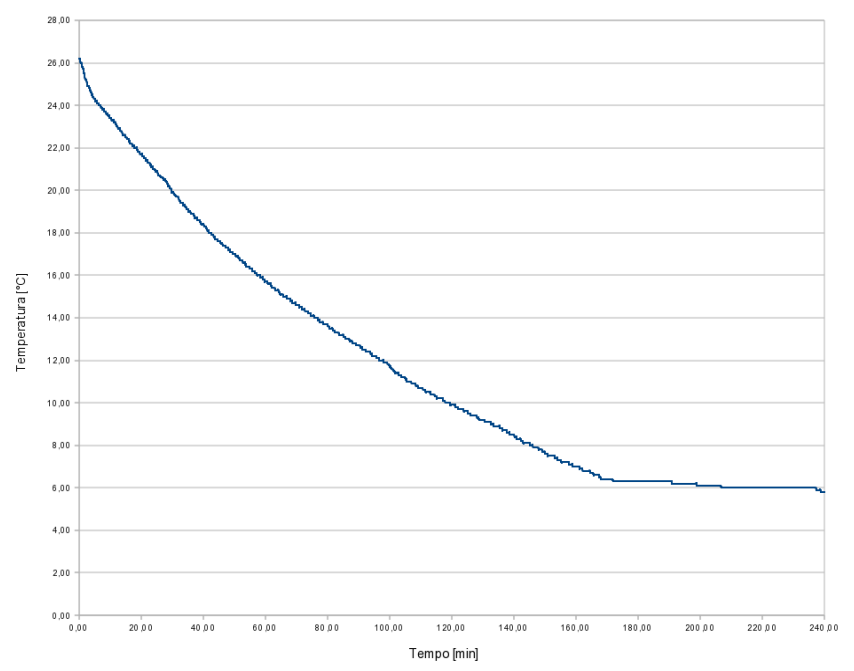

Figura 4 - Temperatura em função do tempo, resultado experimental

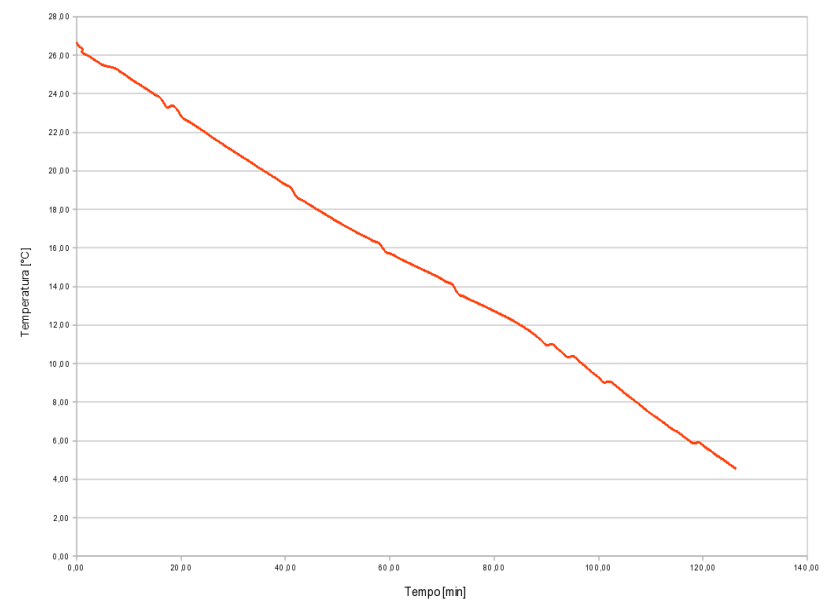

Figura 5 - Temperatura versus tempo, utilizando fluxo $q_{1}=-12000 \mathrm{~W} / \mathrm{m}^{2}$

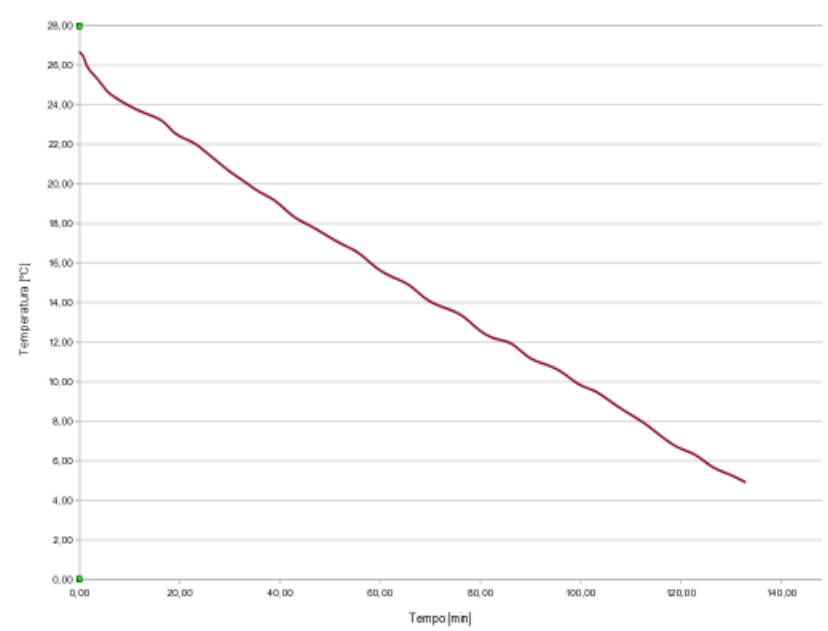

Figura 6. Temperatura versus tempo, utilizando fluxo $q_{2}=-10000 \mathrm{~W} / \mathrm{m}^{2}$ 
A FIGURA 7 reproduz os resultados obtidos a partir das simulações numéricas e os resultados experimentais. Na FIGURA 8 é possível visualizar a topologia do campo de temperaturas dentro do reservatório para o instante de tempo $\mathrm{t}=2.55 \times 10^{3} \mathrm{~s}$. É possível observar uma influência do dispositivo Peltier sobre o campo de temperaturas com estratificação estável, onde as linhas isotérmicas apresentaram um comportamento oscilante ao longo da direção $x$ do domínio, o que não ocorreria no caso da ausência deste dispositivo. Além disso, ocorre um resfriamento do fluido na região próxima ao Peltier e essa massa de fluido resfriada, desce, por diferença de massa específica, na região próxima a superfície lateral esquerda do reservatório, resfriando parte do fluido na região inferior do reservatório.

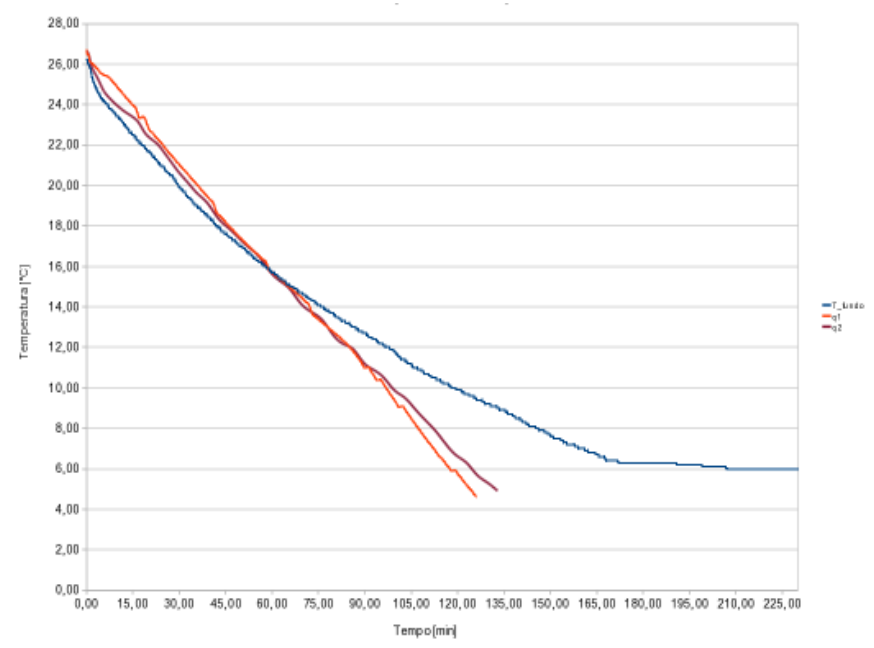

Figura 7 - Comparação entre experimento (linha azul) e simulações

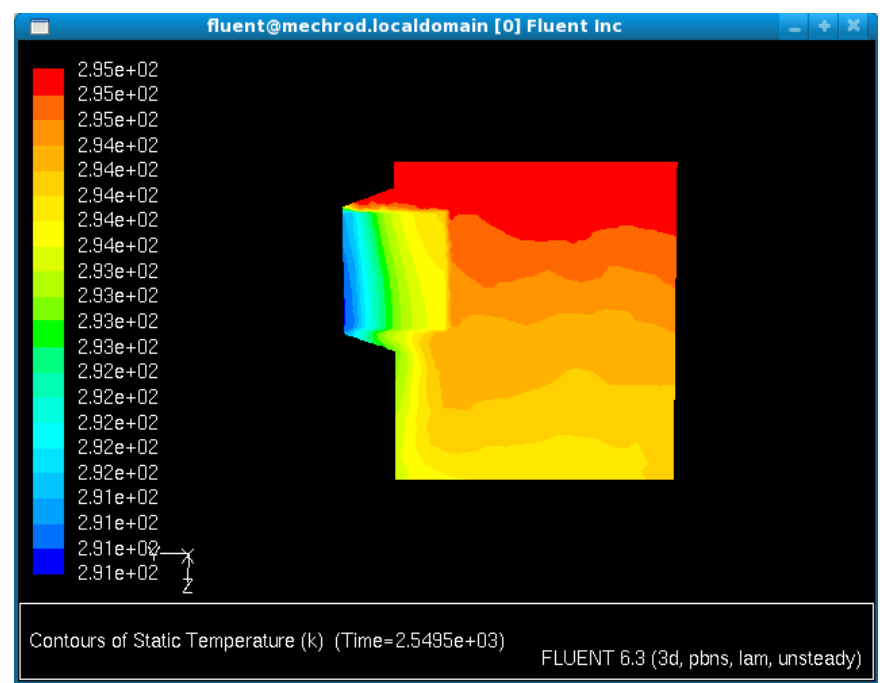

Figura 8 - Gradiente de temperaturas dentro do reservatório após 42 minutos de simulação. Escala de temperatura em Kelvin. 


\section{CONCLUSIONS}

No presente trabalho foi feito o estudo do processo de resfriamento da água, por convecção natural, em um reservatório fechado, através do chamado efeito Peltier ou efeito termoelétrico.

Com relação ao experimento, o único problema encontrado foi o de determinar exatamente a eficiência a ser utilizada para as simulações, já que esta varia, mas de maneira não muito significativa, juntamente com a temperatura da pastilha termoelétrica. Em relação aos outros fatores ambientais do experimento, todos puderam ser devidamente controlados durante a realização do teste.

Em relação à validação, esta foi considerada razoavelmente bem sucedida, pois as duas curvas das simulações apresentaram a mesma tendência de queda gradual na temperatura, apesar de haver alguma diferença quanto aos valores finais do tempo de resfriamento. Acredita-se que uma das razões para essa diferença se deve principalmente ao fato do equipamento não possuir um isolamento perfeito, enquanto a simulação se utiliza desta hipótese.

\section{REFERENCES}

BEJAN, A., Convection Heat Transfer, Jhon Wiley \& Sons, North Caroline, USA, 2004.

DISALVO, F.J., Thermoelectric Cooling and Power Generation, Science, Vol. 285, oㅡ 5428, 1999, pp. 703-706.

FLUENT (Version 6.3.26), ANSYS Inc., 2007.

INCROPERA, F. P.; DEWITT, D. P., BeRGMAN, T. L., LAVINE, A. S.,

Fundamentos de Transferência de Calor e Massa, Editora LTC, 2008.

MASTERFRIO, Manual Técnico Masterfrio, 2008.

RIBERI, F. S., Projeto de uma micro-adega climatizada de baixo custo, Tese de Doutorado, Universidade de São Paulo, 2004.

\section{ACKNOWLEDGEMENTS}

E. D. dos Santos thanks CAPES by his doctorate scholarship and L. A. O. Rocha thanks CNPq for his research grant. 